\title{
BMJ Open Impact of moderate and late preterm birth on neurodevelopment, brain development and respiratory health at school age: protocol for a longitudinal cohort study (LaPrem study)
}

Jeanie Cheong (ID) ,1,2,3 Kate Lillian Iona Cameron (1) , , Deanne Thompson, , ${ }^{1,5,6}$ Peter J Anderson, ${ }^{1,7}$ Sarath Ranganathan, ${ }^{5,8,9}$ Ross Clark, ${ }^{10}$ Benjamin Mentiplay, ${ }^{11}$ Alice Burnett, ${ }^{1,2,5,12}$ Katherine Lee, ${ }^{5,13}$ Lex William Doyle (i) , , ${ }^{1,2,3}$ Alicia J Spittle ${ }^{1,2,4}$

To cite: Cheong J, Cameron KLI, Thompson D, et al. Impact of moderate and late preterm birth on neurodevelopment, brain development and respiratory health at school age: protocol for a longitudinal cohort study (LaPrem study). BMJ Open 2021;11:e044491. doi:10.1136/ bmjopen-2020-044491

- Prepublication history and additional material for this paper are available online. To view these files, please visit the journal online (http://dx.doi. org/10.1136/bmjopen-2020044491).

Received 07 September 2020 Revised 19 November 2020 Accepted 30 December 2020

Deck for updates

(C) Author(s) (or their employer(s)) 2021. Re-use permitted under CC BY-NC. No commercial re-use. See rights and permissions. Published by BMJ.

For numbered affiliations see end of article.

\section{Correspondence to}

Professor Jeanie Cheong; jeanie.cheong@thewomens. org.au

\section{ABSTRACT}

Introduction Children born moderate to late preterm (MLP, 32-36 weeks' gestation) account for approximately $85 \%$ of all preterm births globally. Compared with children born at term, children born MLP are at increased risk of poor neurodevelopmental outcomes. Despite making up the largest group of preterm children, developmental outcomes of children born MLP are less well studied than in other preterm groups. This study aimed to (1) compare neurodevelopmental, respiratory health and brain magnetic resonance imaging (MRI) outcomes between children born MLP and term at 9 years of age; (2) examine the differences in brain growth trajectory from infancy to 9 years between children born MLP and term; and in children born MLP; (3) examine the relationship between brain development and neurodevelopment at 9 years; and (4) identify risk factors for poorer outcomes at 9 years.

Methods and analysis The "LaPrem" (Late Preterm MRI Study) study is a longitudinal cohort study of children born MLP and term controls, born at the Royal Women's Hospital in Melbourne, Australia, between 2010 and 2013. Participants were recruited in the neonatal period and were previously followed up at 2 and 5 years. This 9 -year school-age follow-up includes neuropsychology, motor and physical activities, and lung function assessments, as well as brain MRI. Outcomes at 9 years will be compared between birth groups using linear and logistic regressions. Trajectories of brain development will be compared between birth groups using mixed effects models. The relationships between MRI and neurodevelopmental outcomes, as well as other early predictors of poor 9 -year outcomes, will be explored using linear and logistic regression.

Ethics and dissemination This study was approved by the human research ethics committee at the Royal Children's Hospital, Melbourne, Australia. Study outcomes will be disseminated through peer-reviewed publications, conference presentations and social media.
Strengths and limitations of this study

- This is the first cohort study of Australian children born moderate to late preterm to be assessed at school age with longitudinal MRI brain data.

- Extensive data collected at term equivalent, 2, 5 and 9 years of age will enable us to compare a wide range of important outcomes between birth groups and to better understand developmental trajectories in this population.

- We expect some attrition at the 9-year follow-up and recognise that differences between children who do and do not complete an assessment may introduce bias.

- As this is a hospital-based cohort, the results may not apply to the general population.

- We are unable to assess all important health outcomes due to resource and time constraints.

\section{INTRODUCTION}

Preterm birth ( $<37$ weeks' gestation) accounts for an estimated $10 \%$ of all live birth globally, of which almost $85 \%$ are born moderate to late preterm (MLP: 32-36 weeks' gestation). ${ }^{1}$ In an Australian context, 22000 children were born MLP in 2017, comprising approximately $7 \%$ of all live births. ${ }^{2}$ Despite making up the largest group of preterm births, developmental outcomes of children born MLP are less well understood than preterm groups born earlier (ie, <32 weeks). Emerging research from our team and others over the last decade has highlighted that MLP birth is not as benign as previously thought. ${ }^{3-9}$ Compared with children born at term, MLP children have (1) smaller and less mature brains at term equivalent age using brain $\mathrm{MRI}^{5}$; (2) increased rates of developmental delay and poorer social-emotional development at 
2 years of age ${ }^{3}$; (3) poorer school readiness, worse cognitive performance and greater special education needs $s^{67}$; and (4) increased respiratory morbidity in infancy and early childhood. ${ }^{89}$

Such findings have increased awareness of the morbidities associated with MLP birth and are influencing obstetric decision-making about the timing of MLP births. However, permanent and meaningful effects on obstetric decision-making await reports of clinically important school-age outcomes in an Australian context, which we will obtain with this study. Empowering obstetricians with the knowledge of whether adverse outcomes in the MLP group persist into school age will enable better decision-making regarding the risks and benefits of delivering a baby 'just a bit early'. Knowledge of developmental trajectories for children born MLP, as well as risk factors for poor outcomes at this age, will provide the evidence base for long-term MLP developmental and health surveillance guidelines. Furthermore, an improved understanding of school-age outcomes for children born MLP will have the potential to inform tailored interventions to suit children's specific needs, information that is not available from assessments in the first few years of development.

Early developmental assessments are only moderately predictive of school-age cognitive functioning and academic achievement, with more accurate information obtained through school-age assessments. ${ }^{10}$ Likewise, lung function is better assessed at school age. Currently, it is recommended that very preterm ( $<32$ weeks' gestation) children have regular developmental and health follow-up throughout childhood. ${ }^{11}$ However, similar guidelines are lacking for MLP children, presumably because the few studies describing outcomes during critical periods of development, such as at school age, have not characterised which developmental domains may be affected compared with children born at term. Without this vital information, we cannot design and implement effective interventions to improve outcomes for this population. Moreover, little is known of the risk factors to alert parents and health practitioners to MLP children at greatest risk and who might benefit most from surveillance.

This 9-year follow-up of a pre-existing MLP cohort and term-born controls will allow us to identify specific domains affected by MLP birth, as well as enable us to identify risk factors for poor outcomes, which in turn will inform the design and implementation of effective early interventions to improve outcomes for these vulnerable children.

\section{Aims and hypotheses}

Aim 1

The first aim was to compare outcomes at 9 years between children born MLP and at term, including (1) neurodevelopment outcomes (neuropsychological, academic, behavioural, physical activity and motor function); (2) brain structure and function, using advanced brain MRI measures; and (3) respiratory health and lung function.
Hypothesis 1

At 9 years, compared with term controls, children born MLP will have (1) lower IQ poorer attention, executive function, language, memory and academic achievement, more problems with behaviour, impaired motor function and lower physical activity levels; (2) smaller brain volumes, aberrant cortical morphology and less mature white matter microstructure; and (3) higher rates of wheezing illnesses and more airflow obstruction on lung function testing.

\section{Aim 2}

The second aim was to examine the differences in brain growth trajectory, from infancy to 9 years, between children born MLP and term using longitudinal MRI measures.

\section{Hypothesis 2}

Children born MLP will have slower trajectory of brain growth from infancy to 9 years compared with children born at term.

\section{Aim 3}

The third aim was to examine the relationships between neurodevelopment at 9 years and (1) brain structure at 9 years and (2) the changes in brain measures between term-equivalent age and 9 years in MLP children.

\section{Hypothesis 3}

Aberrant brain development at 9 years, and slower growth and maturation in brain volumes, cortical and white matter brain measures between term-equivalent age and 9 years will be related to poorer developmental outcomes at 9 years for children born MLP.

\section{Aim 4}

The fourth aim was to identify risk factors in pregnancy and early infancy for poorer school age outcomes

(neurodevelopment, brain structure and function and respiratory health) for children born MLP.

\section{Hypothesis 4}

Foetal growth restriction, newborn respiratory problems, smaller brain volumes and less mature white matter microstructure at term-equivalent age, suboptimal neurobehaviour at term equivalent age, and developmental delay and respiratory ill health at 2 years will be associated with poorer school age outcomes. Environmental risk factors (eg, passive smoke exposure and adverse social risk) during pregnancy and early infancy will also be associated with poorer outcomes.

\section{METHODS AND ANALYSIS \\ Protocol details}

Protocol details: V.4. Dated 6 April 2020. Updated to include physically distanced assessment on 26 August 2020. 


\section{Design}

This study is a prospective longitudinal follow-up of an established cohort.

\section{Study population}

The LaPrem study is a cohort study of children born MLP $(n=201)$ and a control group of children born at term ( $\geq 37$ weeks' gestation and $>2499 \mathrm{~g}$ birth weight, $\mathrm{n}=201$ ). Children were recruited in the neonatal period from the Royal Women's Hospital, a large tertiary hospital in Melbourne, Australia, between 2010 and 2013. Children with congenital abnormalities or genetic syndromes known to affect development were excluded. In addition, term-born infants were excluded if they were unwell at birth, received resuscitation, were admitted to the neonatal nursery or were identified as having conditions affecting growth or development.

The LaPrem cohort has previously been assessed at term equivalent age and at 2 and 5 years. Results of these assessments have been published previously. ${ }^{3-5} 12-18$ This protocol paper details the 9-year follow-up of this cohort. All surviving children still enrolled in the LaPrem cohort will be eligible to participate in this study.

\section{Assessment procedure}

Study visits

Families will be contacted by a research nurse and invited to participate in the 9-year assessment. Information about the assessment will be provided through a plain language information and consent form, as well as through a handout designed specifically for the participating children. If parents agree for their child to participate in the 9-year follow-up, they will be asked to sign the participant information and consent form. This consent form has separate, optional consent options for (1) an MRI scan (which is preceded by a session in a mock MRI); (2) a lung function assessment; (3) permission to access the child's National Assessment Programme-Literacy and Numeracy (NAPLAN) results; and (4) permission to access the child's Medicare record, which includes data on outpatient (hospital and health practitioners) and inpatient visits, as well as prescription medications. Parents may consent to some or all of these study aspects on behalf of their child. More information about each of these components is detailed under the specific outcome measures.

Assessments will take place over 2 days, with regular breaks as required to minimise fatigue. All assessors will be blinded to birth group and results of previous assessments. Assessments commenced in June 2019, with data collection proposed to finish in December 2022.

\section{Assessment procedure: COVID-19 adaptations}

The COVID-19 pandemic, which escalated in March 2020 in Victoria, Australia, has necessitated changes to the assessment procedure for this study to ensure, first, that relevant institutional and government guidelines are adhered to and, second, that study participants and assessors are provided with a safe environment. While community transmission of COVID-19 in Victoria, Australia, remains a concern, participants will be offered one of two assessment formats:

1. A face-to-face assessment, with physical distancing measures and hygiene procedures in place to minimise risk of viral transmission.

2. In the case of restrictions prohibiting face-to-face assessments for research purposes, a remote assessment will be offered, in which parts of the cognitive and motor components will be conducted via the video conferencing platform, with the participants in their own home. When these restrictions are lifted, these participants may be offered the opportunity to complete the remaining components of the assessments, including brain MRI and lung function, if possible.

The option of remote assessment may also be offered outside the context of such restrictions to reduce the total time spent onsite or to participants who do not wish to attend the hospital at all due to concerns about viral transmission, or to regional, interstate or international participants who are unable to travel. Further details about adapted assessment procedures are provided in online supplemental material 1.

\section{Outcome measures}

All assessments will be conducted by trained professionals blinded to group allocation and clinical history.

\section{Neuropsychology}

a. General cognitive ability will be assessed using the Wechsler Intelligence Scale for Children-Fifth Edition, Australia and New Zealand. ${ }^{19}$ Ten core subtests will be administered, which will provide an estimate of full-scale IQ as well as composite scores on five domains of cognitive functioning: verbal comprehension, visual spatial, fluid reasoning, working memory and processing speed.

b. Attention will be assessed using subtests from the Test of Everyday Attention for Children, Second Edition. ${ }^{20}$ The five selected subtests include (1) Vigil, a measure of sustained attention where participants keep count of the number of sounds they hear on a recording; (2) Hector, a measure of focused visual attention where participants search for target objects in busy visual arrays; (3) Troy dual task, a more complex divided attention task that combines the previous two tasks, so that the participant searches for visual targets while counting sounds at the same time; (4) simple reaction time, a measure of reaction time where participants press a computer key in response to an on-screen stimulus; and (5) Reds, Blues, Bags, and Shoes, a measure of the participants' ability to rapidly and fluently switch attention between different task requirements.

c. Executive functions will be examined using two tests: (1) the Contingency Naming Test, which measures aspects of executive function such as response inhibition, working memory and mental flexibility, ${ }^{21}$ and 
involves four trials that increase in task complexity, where the participant names shapes and colours according to learnt rules; (2) the Rey Complex Figure Test, which involves reproducing a complicated line drawing, which is assessed for accuracy and strategy. ${ }^{22}$

d. Language skills will be assessed using one subtest from the NEPSY-II, ${ }^{23}$ the Comprehension of Instructions Subtest, which assesses ability to process and execute oral instructions of increasing syntactic complexity. For each item, the child points to stimuli in response to verbal instructions.

e. Memory will be assessed using the California Verbal Learning Test-Children's Version, testing participants' ability to learn a verbally presented word list over multiple learning trials. ${ }^{24}$ The participant's ability to remember the list of words over both short and long time delays will be assessed.

f. Academic achievement will be assessed using the Wechsler Individual Achievement Test-Third Edition, Australia and New Zealand. ${ }^{25}$ The selected subtests will assess reading (single word reading and pseudoword decoding), spelling and math computation. NAPLAN results will provide further data on academic achievement.

\section{Motor and physical activities}

a. Motor function will be assessed using the Movement Assessment Battery for Children, Second Edition, ${ }^{26}$ a gold standard for assessing motor impairment in children 3-16 years of age. The assessment is divided into three subscales: manual dexterity, aiming and catching and balance.

b. Cerebral palsy will be diagnosed based on a neurological examination including loss of motor function, abnormal tone and tendon reflexes, or recorded if the child has previously received a diagnosis. The five-level Gross Motor Function Classification System (GMFCS) will be used to further classify motor function for children with cerebral palsy. The GMFCS is a reliable and widely used scale that classifies children's gross motor function based on self-initiated movement. ${ }^{27}$

c. Physical activity levels will be assessed with a highresolution (13-bit), water resistant to $1.5 \mathrm{~m}$ ankle mounted triaxial accelerometer (Axivity AX3, Newcastle on Tyne, UK) worn for seven consecutive days (including a full weekend) for 24 hours/day. The key outcome measure is mean steps per day, with additional measures including time spent inactive and the time spent at different exercise intensities per day. Ankle-mounted accelerometers have been used previously to assess activity in children with motor impairments, ${ }^{28}$ and the ankle is well established in research as the optimal position for stepping movements across a range of devices. ${ }^{29}$ The monitor will be given to children at the follow-up assessment and returned by post.

d. Physical activity participation will be measured using the Physical Activity Questionnaire for Older Children (PAQ-C) ${ }^{30}$ This questionnaire will be completed by the child during the assessment, with assistance from the assessor, if necessary, using an online survey linked to a Research Electronic Data Capture (REDCap) database. ${ }^{31}$ The PAQ-C is a self-report 7-day recall questionnaire which was developed for children aged 8-14 years and includes a checklist of common sporting and leisure activities, as well as questions about physical activity levels during the school day, after school and on weekends. ${ }^{30}$ The sporting and leisure activity list was updated for the current study to include common activities undertaken by Australian children, with permission from the authors of the outcome measure. The reliability and validity of the PAQ-C have been investigated in several different geographical regions, and studies have demonstrated good reliability and fairmoderate validity with accelerometers. ${ }^{32}$ In addition to the PAQ-C, two brief questions assessing participation in sedentary screen behaviours will be administered. The questions are adapted from the Centers for Disease Control and Prevention 2017 Middle School Youth Risk Behaviour Survey and are as follows:

1. 'On an average school day, how many hours do you watch TV?'

2. On an average school day, how many hours do you play video or computer games or use a computer for something that is not school work? (Count time spent on things such as Xbox, PlayStation, an iPad or other tablet, a smartphone, texting, YouTube, Instagram, Facebook or other social media.) ${ }^{33}$

e. Grip strength will be measured using a hand-held dynamometer. Strength (force in kilogram) will be measured with the child in a seated position, with their elbow flexed at $90^{\circ}$, shoulder adducted into their body and their forearm parallel to the floor. Each child will be given a practice trial, followed by three subsequent trials for each of (1) the child's preferred hand; (2) the child's non-preferred hand; and (3) bimanual, from which the maximum for each condition will be taken. Grip strength is an inexpensive and easily implemented assessment which, in adulthood, can be an indicator of health as poorer grip strength has been associated with functional impairment, ${ }^{34}$ cardiovascular disease and all-cause mortality. ${ }^{35}$

f. Anthropometric measures include height, weight, waist circumference, chest circumference, head circumference, mid-arm circumference and lower limb length, and will be measured according to standard guidelines. In addition, blood pressure, mean arterial pressure and heart rate will be taken at rest using an automated device.

\section{Brain MRI}

A trained research assistant will lead each child through a 'mock' MRI to familiarise the child with the process before undertaking the MRI scan. Only children who are comfortable and able to stay still during the mock MRI procedure will undergo MRI, ensuring high compliance and quality images. Imaging will be undertaken using 


\begin{tabular}{|c|c|}
\hline 1 & $\begin{array}{l}\text { T1- weighted multiecho magnetisation prepared rapid gradient echo (MP-RAGE) images with echo planar image- } \\
\text { navigated prospective motion compensation (TR, } 2550 \text { ms; TE, TE1 } 2.14 \text { ms, TE2 } 3.94 \text { ms, TE3 } 5.77 \text { ms, TE4 } 7.5 \\
\text { ms; IV, } 0.9 \mathrm{~mm}^{3} \text {; TA, 06:47) }\end{array}$ \\
\hline 2 & 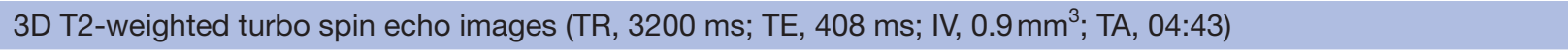 \\
\hline 3 & $\begin{array}{l}\text { Multishell simultaneous multislice echo planar diffusion images (TR, } 3500 \mathrm{~ms} ; \mathrm{TE}, 67 \mathrm{~ms} ; \mathrm{IV}, 2.0 \mathrm{~mm}^{3} ; b=750 \mathrm{~s} / \mathrm{mm}^{2} \\
25 \text { gradient directions; } b=2000 \mathrm{~s} / \mathrm{mm}^{2}, 45 \text { directions; and } b=2800 \mathrm{~s} / \mathrm{mm}^{2}, 60 \text { directions; } 19 \mathrm{~b}=0 \mathrm{~s} / \mathrm{mm}^{2} ; \mathrm{multiband}^{2} \\
\text { acceleration factor } 2 ; \mathrm{TA}, 10: 59) \text { with matching reverse-phase encoding images (TA, 01:40) }\end{array}$ \\
\hline 4 & 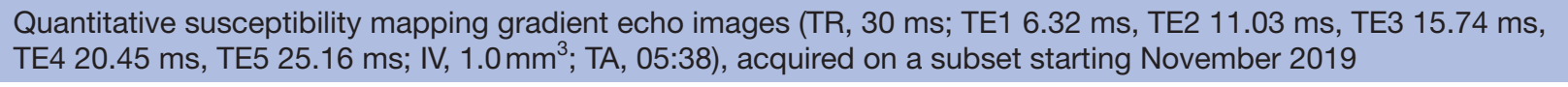 \\
\hline & $\begin{array}{l}\text { Multiecho gradient recalled echo planar resting state functional MRI images with prospective acquisition correction } \\
\text { (TR, } 1500 \mathrm{~ms} \text {; TE, } 33 \mathrm{~ms} \text {; IV, } 2.5 \mathrm{~mm}^{3} \text {; multiband acceleration factor } 3 \text {; total TA, 13:04, in two consecutive } \\
\text { acquisitions of TA, 06:32, for compliance) with matching reverse-phase encoding images (TA, 0:56) }\end{array}$ \\
\hline
\end{tabular}

IV, isotropic voxel; TA, time of acquisition; TE, echo time; TR, repetition time.

a 3-Tesla Siemens MAGNETOM Prisma scanner at the Royal Children's Hospital MRI Centre using sequences listed in table 1 . Children will be scanned within 2 weeks of their neuropsychological assessment. We will quantify brain tissue volumes for over 100 brain regions and structures, as well as cortical morphology, including surface area, thickness, curvature, folding and sulcal depth for around 68 cortical regions, using the validated software FreeSurfer V.7. ${ }^{36}$ A mathematical ratio of the $T_{1}$ to $T_{2}$ images will be calculated to derive myelin maps, ${ }^{37}$ providing a sensitive measure of myelination throughout the entire brain. Diffusion modelling techniques will be used to measure microstructural complexity, including traditional diffusion tensor imaging, ${ }^{38}$ as well as more advanced models: (1) the novel Spherical Mean Technique, which can estimate diffusivity and neurite density without influence from crossing fibres ${ }^{39}$; and (2) fixelbased analyses, which can quantify the apparent fibre density of individual fibres within each diffusion image voxel. ${ }^{40}$ Seventy-two major white matter tracts will be virtually dissected using TractSeg, a novel convolutional neural network-based approach that directly segments tracts in the field of fibre orientation distribution function peaks with high accuracy, without using tractography, image registration or parcellation. ${ }^{41}$ We will use modern mathematical methods of graph theory and networkbased statistics ${ }^{42}$ to determine how different parts of the brain interconnect both structurally (using constrained spherical deconvolution ${ }^{43}$ ) and functionally (using resting state functional MRI). The sensitive MRI analysis techniques will be useful for determining brain developmental maturity and hold great promise for uncovering the subtler brain abnormalities associated with MLP birth.

\section{Lung function}

Lung function (detailed in table 2) will be measured in the Respiratory Function Laboratory. Spirometry will be measured according to standard guidelines of the American Thoracic Society ${ }^{44}$ and expressed primarily as z-scores. ${ }^{45}$ Plethysmography to assess lung volumes and measurement of gas transfer are standardised tests routinely used in clinical practice. The Lung Clearance Index is an innovative test that measures ventilation inhomogeneity; it is more sensitive than spirometry in detecting early airway dysfunction, and it has reference data available. ${ }^{46}$

\section{Parent questionnaires}

Parents/caregivers will be asked to complete several questionnaires (table 3) via online surveys which are linked to a REDCap database. Questionnaires, which are to be completed by the primary caregiver, include four measures of child behaviour, parent-reported assessments of child motor skills and respiratory symptoms, as well as demographic data to determine social risk. In addition, both parents will be asked to complete the

Table 2 Lung function variables

\begin{tabular}{ll}
\hline Lung function & Variable measured \\
\hline Airflow & FEV $_{1}$, FEF25\%-75\% \\
Lung volumes & FVC, TLC, RV \\
Reversibility & Bronchodilator to determine how much airway obstruction is reversible. \\
Gas exchange & $\mathrm{D}_{\text {Lco }}$ : measures alveolar-capillary membrane pathology \\
\hline Ventilation inhomogeneity & Lung Clearance Index, second (airway conductance) \\
\hline
\end{tabular}

$D_{L c o}$, diffusing capacity of the lung for carbon monoxide; FEF25\%-75\%, forced mid-expiratory flow; $F E V_{1}$, forced expiratory volume in one second; FVC, forced vital capacity; RV, residual volume; TLC, total lung capacity. 
Table 3 Parent questionnaires

\begin{tabular}{|c|c|}
\hline Questionnaire & Description \\
\hline $\begin{array}{l}\text { Developmental Coordination Disorder } \\
\text { Questionnaire }\end{array}$ & $\begin{array}{l}\text { Parent-reported measure designed to identify subtle motor problems in } \\
\text { children; construct validity has been established, and cut-off scores to } \\
\text { determine risk of motor impairment are available for this age group. }\end{array}$ \\
\hline Strengths and Difficulties Questionnaire ${ }^{50}$ & $\begin{array}{l}\text { Parent-reported, well-validated measure that assesses children's overall } \\
\text { behaviour problems, emotional symptoms, hyperactivity/attention, peer } \\
\text { relationship problems and prosocial behaviour }\end{array}$ \\
\hline $\begin{array}{l}\text { Behaviour Rating Inventory of Executive } \\
\text { Functioning, Second Edition }{ }^{51}\end{array}$ & $\begin{array}{l}\text { Parent-reported measure designed to assess children's executive } \\
\text { function behaviours in the home environment }\end{array}$ \\
\hline $\begin{array}{l}\text { Social Communication Questionnaire-Current } \\
\text { Version }^{52}\end{array}$ & $\begin{array}{l}\text { Parent-reported measure used to evaluate children's communication } \\
\text { skills and social functioning }\end{array}$ \\
\hline $\begin{array}{l}\text { ADHD Rating Scale- } 5 \text { for Children and } \\
\text { Adolescents }{ }^{53}\end{array}$ & $\begin{array}{l}\text { Parent-reported measure to assess potential symptoms of ADHD. The } \\
\text { ADHD Rating Scale- } 5 \text { is a valid and reliable instrument that is widely used } \\
\text { by practitioners in screening, diagnosis and treatment evaluation. }\end{array}$ \\
\hline $\begin{array}{l}\text { The International Study of Asthma and Allergies in } \\
\text { Childhood Core Questionnaire }\end{array}$ & $\begin{array}{l}\text { Parent-reported measure to assess for symptoms of asthma, allergic } \\
\text { rhinitis and eczema }\end{array}$ \\
\hline Social Risk Index ${ }^{55}$ & $\begin{array}{l}\text { Assesses six aspects of social risk, including family structure, education } \\
\text { of primary caregiver, occupation of primary income earner, employment } \\
\text { status of primary income earner, language spoken at home and maternal } \\
\text { age at birth. This measure has been used successfully in previous studies } \\
\text { considering children born preterm. }{ }^{56}\end{array}$ \\
\hline The Hospital Anxiety and Depression Scale ${ }^{47}$ & $\begin{array}{l}\text { To be completed by both caregivers, this questionnaire is a self-rating } \\
\text { scale that measures anxiety and depression in both hospital and } \\
\text { community settings }\end{array}$ \\
\hline
\end{tabular}

ADHD, attention deficit hyperactivity disorder.

Hospital Anxiety and Depression Scale. ${ }^{47}$ Parents who report a moderate-severe level of symptoms (score above 11) will be contacted, and relevant support services will be suggested to them. If they are unable to be contacted by phone within a reasonable time frame, a handout of contact information for available local supports will be provided by email.

\section{Assessment feedback for participants}

All participants will receive written results from all assessments in a formal report. Results will be articulated in terms of performance ranges (ie, below average, average and above average) for neuropsychology and motor assessments. The report will include contact details for the study team, and families will be encouraged to contact the team if they wish to discuss the information in the report. If there are assessment results that require clinical follow-up, a researcher will contact the family directly to discuss the findings and options for referral to appropriate services as indicated.

\section{Sample size}

The sample size is determined by the size of the existing cohort $(n=402)$. Based on our past experience of assessing many similar age cohorts, we conservatively estimate an overall follow-up rate of $90 \%$ at the 9-year assessment; this will result in $180 \mathrm{MLP}$ and 180 controls. There are 27 pairs of twins in the MLP group (27\% of participants) and none in the term control group. Assuming an intraclass correlation coefficient of 0.6 between outcomes for twins and that $27 \%$ of those who attend the follow-up are twins $(n=48)$, we will have an effective sample size of 155 MLP (based on a design effect of 1.16). Samples of 155 MLP and 180 controls will enable us to detect differences between the groups as small as 0.31 SD with $80 \%$ power (based on a two-sided test with $\alpha=0.05$ ). For most outcomes that are measured continuously, a difference of $0.31 \mathrm{SD}$ between groups would be clinically important and is a difference that we could potentially observe. For proportions, if the event rate is $50 \%$ in the controls, we will have $80 \%$ power to detect differences of $\pm 16 \%$. As the event rate moves away from $50 \%$, we will be able to detect smaller absolute differences between the two groups with $80 \%$ power, which are again clinically important differences, and differences that we could potentially observe.

\section{Data management}

All neuropsychology, motor and lung function outcome data will be entered into an electronic REDCap database by a member of the research team. Parent-completed questionnaires, as well as the PAQ-C, which is completed by the child, will be completed as online surveys linked to the REDCap database. Data will be stored in a secure data storage facility based at the Murdoch Children's Research Institute (MCRI) and backed up daily onto a central server. Data will be stored until the youngest participant reaches 25 years of age. Participant's deidentified MRI 
data will be stored and backed up in the secure developmental imaging facility initially on-site at MCRI and later archived at a secure off-site facility. The Quantitative susceptibility mapping (QSM) MRI images will be analysed in collaboration with the Florey Institute of Neuroscience and Mental Health, which has previously applied these techniques in neurodegeneration research. ${ }^{48} \mathrm{~A}$ mutual transfer agreement will be set up prior to commencement of analysis. Any modifications to the approved study protocol will need a formal amendment to the research and ethics committee of the Royal Children's Hospital.

\section{Statistical analysis}

\section{Aim 1}

Outcomes will be compared between MLP and term-born controls using linear (continuous) and logistic (binary) regression, both unadjusted and adjusted for potential confounding variables, including gestation at birth, sex and social risk. Models will be fitted using generalised estimating equations (GEEs) and reported with robust (sandwich) estimates of SEs to allow for clustering of siblings within a family.

\section{Aim 2}

Longitudinal differences in brain MRI measures from term equivalent age to 9 years will be compared between MLP and controls using mixed effects models, including a fixed effect of age, group and the interaction between age and group, and a random effect to allow for the repeated observations within an individual.

\section{Aim 3}

To determine the relationships between imaging metrics and neurodevelopmental outcomes, we will use linear and logistic regressions (fitted with GEEs), with adjustment for social and environmental factors, including gestation at birth, sex and social risk, to determine the relationships over and above these factors.

\section{Aim 4}

Linear and logistic regressions (fitted using GEEs) will be used to assess predictors (brain MRI, including quantitative and qualitative measures at term equivalent age, gestation at birth, sex, birth weight z-score and 2 year neurodevelopment) of outcomes at 9 years. Initially, potential predictors will be explored using univariable regression for each predictor-outcome combination, before combining predictors into a multivariable model for each outcome to assess independent predictors. Social factors (maternal education and occupation of primary caregiver) will be assessed as modifiers in these relationships by the inclusion of interaction terms between the predictors and the social factor to the multivariable regression models.

\section{Patient and public involvement}

The development of the research question and outcome measures are based on the findings of our previous research into the health and developmental outcomes of MLP from infancy to 2 years, and other research. ${ }^{3-9}$ The importance of long-term outcomes was discussed in a workshop sponsored by our National Health and Medical Research Council-funded Centre of Research Excellence (CRE) in Newborn Medicine, which included parent representatives. ${ }^{11}$ While there was no direct involvement of MLP individuals or their families in the design of the study, the CRE in the Newborn Medicine Consumer Advisory Group, which includes individuals born preterm and their families, will be involved in designing a knowledge translation plan to disseminate study findings.

\section{Ethics and dissemination}

This study has ethical approval from the human research ethics committee at the Royal Children's Hospital, Melbourne, Australia. Study outcomes will be presented through peer-reviewed publications and conference presentations. In addition, our results will be disseminated to key stakeholders, including clinicians, parents and teachers, facilitated by our CRE in the Newborn Medicine Consumer Advisory Group and partnerships with parent support groups.

\section{Author affiliations}

${ }^{1}$ Clinical Sciences, Murdoch Children's Research Institute, Parkville, Victoria, Australia

${ }^{2}$ Newborn Research, Royal Women's Hospital, Parkville, Victoria, Australia

${ }^{3}$ Department of Obstetrics and Gynaecology, The University of Melbourne, Melbourne, Victoria, Australia

${ }^{4}$ Department of Physiotherapy, The University of Melbourne, Melbourne, Victoria, Australia

${ }^{5}$ Department of Paediatrics, The University of Melbourne, Melbourne, Victoria, Australia

${ }^{6}$ Florey Institute of Neuroscience and Mental Health, Melbourne, Victoria, Australia ${ }^{7}$ Monash Institute of Cognitive and Clinical Neurosciences, Monash University, Melbourne, Victoria, Australia

${ }^{8}$ Respiratory Medicine, Royal Children's Hospital, Melbourne, Victoria, Australia ${ }^{9}$ Respiratory Diseases, Murdoch Children's Research Institute, Melbourne, Victoria, Australia

${ }^{10}$ Research Health Institute, University of the Sunshine Coast, Sunshine Coast, Queensland, Australia

${ }^{11}$ La Trobe Sport and Exercise Medicine Research Centre, La Trobe University, Melbourne, Victoria, Australia

${ }^{12}$ Department of Neonatal Medicine, The Royal Children's Hospital, Melbourne, Victoria, Australia

${ }^{13}$ Clinical Epidemiology and Biostatistics, Murdoch Children's Research Institute, Melbourne, Victoria, Australia

Twitter Kate Lillian Iona Cameron @kate_cameron and Benjamin Mentiplay @ MentiplayB

Contributors JC conceived and designed the study, drafted and critically revised the manuscript and gave the final approval of the version to be published. KLIC drafted and critically revised the manuscript and gave final approval of the version to be published. DT, PJA, SR, RAC, BM, AB, KL, LWD and AJS conceived and designed the study, critically revised the manuscript and gave the final approval of the version to be published.

Funding This study is supported by grants from the National Health and Medical Research Council of Australia (project grant \#1161305), Centre of Research Excellence (\#1153176), Career Development Fellowship (\#1160003 to DT, 1127984 to KL, \#1108714 to AJS, Leadership Fellowship \#1176077 to PJA), Medical Research Future Fund (Career Development Fellowship \#1141354 to JC) and the Victorian Government's Operational Infrastructure Support Program.

Competing interests None declared.

Patient consent for publication Not required. 
Provenance and peer review Not commissioned; peer reviewed for ethical and funding approval prior to submission.

Supplemental material This content has been supplied by the author(s). It has not been vetted by BMJ Publishing Group Limited (BMJ) and may not have been peer-reviewed. Any opinions or recommendations discussed are solely those of the author(s) and are not endorsed by BMJ. BMJ disclaims all liability and responsibility arising from any reliance placed on the content. Where the content includes any translated material, BMJ does not warrant the accuracy and reliability of the translations (including but not limited to local regulations, clinical guidelines, terminology, drug names and drug dosages), and is not responsible for any error and/or omissions arising from translation and adaptation or otherwise.

Open access This is an open access article distributed in accordance with the Creative Commons Attribution Non Commercial (CC BY-NC 4.0) license, which permits others to distribute, remix, adapt, build upon this work non-commercially, and license their derivative works on different terms, provided the original work is properly cited, appropriate credit is given, any changes made indicated, and the use is non-commercial. See: http://creativecommons.org/licenses/by-nc/4.0/.

\section{ORCID iDs}

Jeanie Cheong http://orcid.org/0000-0001-5901-0455

Kate Lillian Iona Cameron http://orcid.org/0000-0001-5447-594X

Lex William Doyle http://orcid.org/0000-0002-7667-7312

\section{REFERENCES}

1 Chawanpaiboon S, Vogel JP, Moller A-B, et al. Global, regional, and national estimates of levels of preterm birth in 2014: a systematic review and modelling analysis. Lancet Glob Health 2019;7:e37-46.

2 Australian Institute of Health and Welfare. Australia's mothers and babies 2017-in brief. Canberra: AlHW, 2019.

3 Cheong JL, Doyle LW, Burnett AC, et al. Association between moderate and late preterm birth and neurodevelopment and Social-Emotional development at age 2 years. JAMA Pediatr 2017;171:e164805

4 Kelly CE, Cheong JLY, Gabra Fam L, et al. Moderate and late preterm infants exhibit widespread brain white matter microstructure alterations at term-equivalent age relative to term-born controls. Brain Imaging Behav 2016;10:41-9.

5 Walsh JM, Doyle LW, Anderson PJ, et al. Moderate and late preterm birth: effect on brain size and maturation at term-equivalent age. Radiology 2014;273:232-40.

6 Bentley JP, Roberts CL, Bowen JR, et al. Planned birth before 39 weeks and child development: a population-based study. Pediatrics 2016;138:e20162002.

7 Chyi LJ, Lee HC, Hintz SR, et al. School outcomes of late preterm infants: special needs and challenges for infants born at 32 to 36 weeks gestation. J Pediatr 2008;153:25-31.

8 Vrijlandt EJLE, Kerstjens JM, Duiverman EJ, et al. Moderately preterm children have more respiratory problems during their first 5 years of life than children born full term. Am J Respir Crit Care Med 2013;187:1234

9 Harju M, Keski-Nisula L, Georgiadis L, et al. The burden of childhood asthma and late preterm and early term births. J Pediatr 2014;164:295-9.

10 Roberts G, Anderson PJ, Doyle LW, et al. The stability of the diagnosis of developmental disability between ages 2 and 8 in a geographic cohort of very preterm children born in 1997. Arch Dis Child 2010;95:786-90.

11 Doyle LW, Anderson PJ, Battin M, et al. Long term follow up of high risk children: who, why and how? BMC Pediatr 2014;14:1.

12 Cheong JLY, Thompson DK, Spittle AJ, et al. Brain volumes at TermEquivalent age are associated with 2-year neurodevelopment in moderate and late preterm children. J Pediatr 2016;174:91-7.

13 Spittle AJ, Walsh JM, Potter C, et al. Neurobehaviour at termequivalent age and neurodevelopmental outcomes at 2 years in infants born moderate-to-late preterm. Dev Med Child Neurol 2017;59:207-15.

14 Eeles AL, Walsh JM, Olsen JE, et al. Continuum of neurobehaviour and its associations with brain MRI in infants born preterm. BMJ Paediatr Open 2017;1:e000136.

15 Kelly CE, Thompson DK, Cheong JL, et al. Brain structure and neurological and behavioural functioning in infants born preterm. Dev Med Child Neurol 2019;61:820-31.

16 Thompson DK, Kelly CE, Chen J, et al. Characterisation of brain volume and microstructure at term-equivalent age in infants born across the gestational age spectrum. Neuroimage Clin 2019;21:101630.

17 Thompson DK, Kelly CE, Chen J, et al. Early life predictors of brain development at term-equivalent age in infants born across the gestational age spectrum. Neuroimage 2019;185:813-24

18 Kelly CE, Thompson DK, Spittle AJ, et al. Regional brain volumes, microstructure and neurodevelopment in moderate-late preterm children. Arch Dis Child Fetal Neonatal Ed 2020;105:593-9.

19 Wechsler D. Wechsler intelligence scale for children, 5th edition: Australian and New Zealand standardised edition (WISC-V A\&NZ). Bloomington, MN: Pearson, 2016.

20 Manly T. Test of everyday attention for children, 2nd edition (TEACh2). London: Pearson, 2016.

21 Anderson P, Anderson V, Northam E. Standardization of the contingency naming test (CNT) for school-aged children: a measure of reactive flexibility. Clinical neuropsychological assessment;12001:385-406.

22 Anderson P, Anderson V, Garth J. Assessment and development of organizational ability: the Rey complex figure organizational strategy score (RCF-OSS). Clin Neuropsychol 2001;15:81-94.

23 Korkman M, Kirk U, Kemp S. NEPSY-II. Bloomington, MN: Pearson, 2007.

24 Delis D, Kramer J, Kaplan E, et al. California verbal learning test Children's Version (CVLT-C): Manual. San Antonio: The Psychological Corporation, 1994.

25 Joshua N, Wilson C, Bendrup N. Wechsler individual achievement test - 3rd edition Australia and New Zealand (WIAT-IIIA\&NZ). Sydney, NSW: The Psychological Corporation, 2016.

26 Henderson SE, Sugden DA, Barnett AL. Movement assessment battery for children-2 second edition (movement ABC-2). London, UK: The Psychological Corporation, 2007.

27 Palisano R, Rosenbaum P, Walter S, et al. Development and reliability of a system to classify gross motor function in children with cerebral palsy. Dev Med Child Neurol 1997;39:214-23.

28 Balemans ACJ, van Wely L, Becher JG, et al. Longitudinal relationship among physical fitness, Walking-Related physical activity, and fatigue in children with cerebral palsy. Phys Ther 2015;95:996-1005.

29 Korpan SM, Schafer JL, Wilson KC, et al. Effect of ActiGraph GT3X+ position and algorithm choice on step count accuracy in older adults. J Aging Phys Act 2015;23:377.

30 Kowalski K, Crocker P, Donen R. The physical activity questionnaire for older children (PAQ-C) and adolescents (PAQ-A) manual. Saskatoon, Canada: College of Kinesiology, University of Saskatchewan

31 Harris PA, Taylor R, Thielke R, et al. Research electronic data capture (REDCap)--a metadata-driven methodology and workflow process for providing translational research informatics support. J Biomed Inform 2009;42:377-81.

32 Gobbi E, Elliot C, Varnier M, et al. Psychometric properties of the physical activity questionnaire for older children in Italy: testing the validity among a general and clinical pediatric population. PLoS One 2016;11:e0156354

33 Centers for Disease Control and Prevention (CDC). 1995-2017 middle school youth risk behavior survey data, 2019. Available: http://nccd.cdc.gov/youthonline/

34 Snih SA, Markides KS, Ottenbacher KJ, et al. Hand grip strength and incident ADL disability in elderly Mexican Americans over a sevenyear period. Aging Clin Exp Res 2004;16:481-6.

35 Leong DP, Teo KK, Rangarajan S, et al. Prognostic value of grip strength: findings from the prospective urban rural epidemiology (pure) study. Lancet 2015;386:266-73.

36 Fischl B. FreeSurfer. Neuroimage 2012;62:774-81.

37 Ganzetti M, Wenderoth N, Mantini D. Whole brain myelin mapping using T1- and T2-weighted MR imaging data. Front Hum Neurosci 2014;8:671

38 Behrens TEJ, Woolrich MW, Jenkinson M, et al. Characterization and propagation of uncertainty in diffusion-weighted MR imaging. Magn Reson Med 2003;50:1077-88.

39 Kaden E, Kelm ND, Carson RP, et al. Multi-Compartment microscopic diffusion imaging. Neuroimage 2016;139:346-59.

40 Raffelt DA, Tournier J-D, Smith RE, et al. Investigating white matter fibre density and morphology using fixel-based analysis. Neuroimage 2017;144:58-73.

41 Wasserthal J, Neher P, Maier-Hein KH. TractSeg - Fast and accurate white matter tract segmentation. Neuroimage 2018;183:239-53.

42 Zalesky A, Fornito A, Bullmore ET. Network-Based statistic: identifying differences in brain networks. Neuroimage 2010;53:1197-207.

43 Jeurissen B, Leemans A, Tournier J-D, et al. Investigating the prevalence of complex fiber configurations in white matter tissue 
with diffusion magnetic resonance imaging. Hum Brain Mapp 2013;34:2747-66.

44 American Thoracic Society. Standardization of spirometry. Am J Respir Crit Care Med 1995;152:1107-36.

45 Quanjer PH, Stanojevic S, Cole TJ, et al. Multi-Ethnic reference values for spirometry for the 3-95-yr age range: the global lung function 2012 equations. Eur Respir J 2012;40:1324-43.

46 Lum S, Stocks J, Stanojevic S, et al. Age and height dependence of lung clearance index and functional residual capacity. Eur Respir $J$ 2013;41:1371-7.

47 Snaith R, Zigmond A. The hospital anxiety and depression scale (HADS). Brentford: GL Education, 1994.

48 Ayton S, Fazlollahi A, Bourgeat P, et al. Cerebral quantitative susceptibility mapping predicts amyloid- $\beta$-related cognitive decline. Brain 2017:140:2112-9.

49 Wilson BN, Crawford SG, Green D, et al. Psychometric properties of the revised developmental coordination disorder questionnaire. Phys Occup Ther Pediatr 2009;29:182-202.
50 Goodman A, Goodman R. Strengths and difficulties questionnaire as a dimensional measure of child mental health. $J$ Am Acad Child Adolesc Psychiatry 2009;48:400-3.

51 Gioia G, Isquith P, Guy S. BRIEF-2 - behavior rating inventory of executive function second edition. Professional manual. Odessa, FL: Psychological Assessment Resources Inc, 2015.

52 Rutter M, Bailey A, Lord C. Social communication questionnaire (SCQ. Torrance, CA: WPS, 2003.

53 DuPaul GJ, Power TJ, Anastopoulos AD, et al. Adhd rating scale-5 for children and adolescents. New York: The Guildford Press, 2016.

54 Ellwood P, Asher MI, Beasley R, et al. The International study of asthma and allergies in childhood (Isaac): phase three rationale and methods. Int J Tuberc Lung Dis 2005;9:10-16.

55 Pace CC, Spittle AJ, Molesworth CM-L, et al. Evolution of depression and anxiety symptoms in parents of very preterm infants during the newborn period. JAMA Pediatr 2016;170:863.

56 Spittle AJ, Treyvaud K, Lee KJ, et al. The role of social risk in an early preventative care programme for infants born very preterm: a randomized controlled trial. Dev Med Child Neurol 2018;60:54-62. 